\title{
Diagnostic des procédés de fabrication du fromage blanc traditionnel «mashanza» dans les zones productrices du Sud-Kivu
}

\author{
Birali Mwamini Jennifer1, Walangululu Jean'1et Sumbu Zola² \\ 1 Université Catholique de Bukavu (UCB), BP 02, Cyangugu, Rwanda \\ Université de Kinshasa (UNIKIN), BP 117, Kinshasa II, RD Congo. \\ *Corresponding Author Email: birali.jennifer@gmail.com
}

Original submitted in on $6^{\text {th }}$ March 2019. Published online at www.m.elewa.org/journals/ on $30^{\text {th }}$ June 2019 https://dx.doi.org/10.4314/jab.v138i1.2

\section{RÉSUMÉ}

Objectif : Le «Mashanza» est un fromage frais au lait de vache fabriqué à l'est de la RD. Congo possédant une valeur nutritionnelle et culturelle importantes, mais dont les procédés de fabrication et la qualité ne sont pas suffisamment maîtrisés. La présente étude vise à réaliser un diagnostic des procédés usuels de fabrication du «Mashanza».

Méthodologie et résultats : Une enquête a été réalisée sur un échantillon de 132 producteurs à Kabare (20), Kalehe (18), Uvira (45) et Walungu (49). Cinq procédés ont été trouvés, dont les plus fréquents suivent les étapes suivantes: collecte du lait -mise en cuve - inoculation - fermentation - égouttage et conditionnement. Le rendement fromager pour $100 \mathrm{~kg}$ de lait est de $24 \pm 11 \mathrm{Kg}$ à Kabare, $24 \pm 10 \mathrm{Kg}$ à Kalehe, $21 \pm 8 \mathrm{Kg}$ à Uvira et $23 \pm 6 \mathrm{Kg}$ à Walungu. Selon les producteurs, le "Mashanza» possède une consistance ferme ou molle, un goût neutre, sucré ou aigre, une odeur de lait ou de fermentation; la couleur variant du blanc au blanc-jaunâtre. En l'absence de la chaîne du froid, la durée de conservation dépasse rarement 7 jours. Les équipements les plus utilisés sont les cuves de fermentation en plastique avec une perforation à la base ou muni d'un robinet pour l'égouttage du lactosérum. Pour le conditionnement, le «Mashanza » est mis dans les sachets plastiques, les feuilles de bananier, les assiettes, pour la vente au détail et dans des seaux avec couvercle pour la vente en gros.

Conclusion et application des résultats : La variabilité des matières premières, l'empirisme des procédés en termes d'étapes et de timing, la non maîtrise des conditions de mise en œuvre et le mode de conditionnement prédisposent à l'obtention des produits de qualité variable et de durée de conservation très limitée. Les résultats obtenus constituent les données de base permettant d'orienter les études ultérieures en vue de l'amélioration et de la normalisation de la qualité de "Mashanza», compte tenu de la rareté d'études sur ce produit. Ils peuvent également aider les chercheurs dans l'optimisation des procédés et de la qualité, à l'identification des risques et orienter les fabricants dans le choix des meilleurs procédés et des pratiques afin d'offrir aux consommateurs des produits de meilleure qualité.

Mots-clés : fromage frais, Mashanza, diagnostic, procédés, qualité, Sud-Kivu 
Study on the manufacturing processes of traditional white cheese "mashanza" in the South-Kivu.

\section{ABSTRACT}

Objective: "Mashanza" is a fresh cow's milk cheese made in eastern DR Congo and with significant nutritional and cultural value, but whose manufacturing processes and quality are not sufficiently controlled. The aim of the study is to carry out a diagnosis of processes for producing "Mashanza "

Methodology and results: A survey was conducted on a sample of 132 producers in Kabare (20), Kalehe (18), Uvira (45) and Walungu (49). Five processes were found, the most frequent follows the following steps: collection of milk - filling of the tank- inoculation - fermentation - draining and conditioning. The cheese yield per $100 \mathrm{~kg}$ of milk is $24 \pm 11 \mathrm{~kg}$ in Kabare, $24 \pm 10 \mathrm{~kg}$ in Kalehe, $21 \pm 8 \mathrm{~kg}$ in Uvira and $23 \pm$ $6 \mathrm{~kg}$ in Walungu. According to the producers, "Mashanza" has a firm or soft consistency, a neutral, sweet or sour taste, a smell of milk or fermentation; the color varies from white to yellowish-white. In the absence of the cold chain, the shelf life rarely exceeds 7 days. The most used equipment is the plastic fermentation tank with a perforation at the base or equipped with a tap for draining the whey. For packaging, the "Mashanza" is put in plastic bags, banana leaves, plates, for retail sale and in buckets with lids for wholesale.

Conclusion and application of results: The variability of the raw materials, the empiricism of processes in terms of steps and timing, the lack of control of implementation conditions and the mode of conditioning may influence variable quality products and induce very limited shelf life. The results obtained constitute the basic data to guide further studies for the improvement and standardization of the quality of "Mashanza" given the scarcity of studies on this product. They can also help researchers in process and quality optimization, identify risks and guide manufacturers in choosing the best processes and practices to provide consumers with higher quality products.

Keywords: fresh cheese, Mashanza, diagnosis, processes, quality, South-Kivu

\section{INTRODUCTION}

Plusieurs types de produits laitiers fermentés existent à travers le monde (Stanley et Tamime, cités par Mechai et al., 2014). Les fromages sont des formes de conservation et de report ancestral de la matière utile dont les qualités nutritionnelles et organoleptiques sont appréciées par l'homme (Jeantet et al., 2008). La production des fromages permet d'allonger la durée de vie et d'augmenter la plus-value du lait (Tchobo et al., 2004). Toutefois, malgré l'essor que connait le secteur fromager dans les pays industrialisés, dans les pays en développement, la fabrication du fromage garde encore un caractère traditionnel (Capo-Chichi, cité par Kora, 2005). En RD Congo, le fromage frais appelé "Mashanza» est un produit traditionnel typique du Sud-Kivu (Prajapati, 2011). Son origine se situe autour de Kabare, un des territoires du Sud-Kivu et dont la production s'est étendue vers d'autres zones. Le lactosérum, appelé «Magunjo», est utilisé comme ferment et consommé comme boisson (FAO, 1990). La figure 1 montre le
« mashanza » produit à Bweghera, dans la plaine de la Ruzizi, au Sud-Kivu.

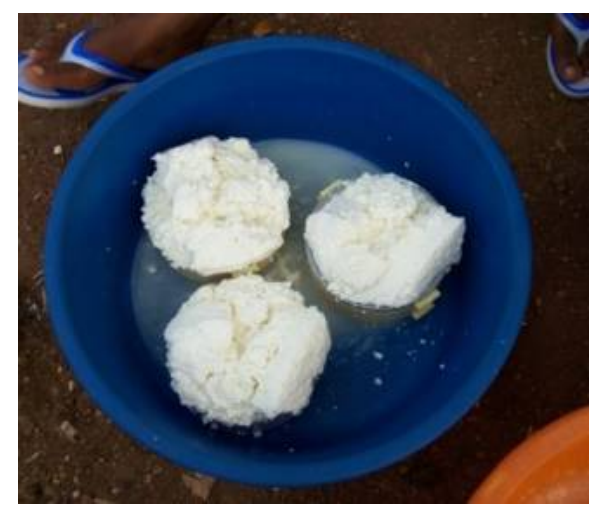

Figure 1: Fromage "Mashanza » destiné à la vente, à Bweghera

Actuellement, on dénombre plusieurs producteurs dans des zones différentes : Kabare, Kalehe, Uvira et Walungu et au-delà du Sud-Kivu (Nord-Kivu, Ituri, à l'est du Congo et le Rwanda), 
correspondant à des zones agro-écologiques distinctes. Les procédés de fabrication utilisés varient suivant la localisation donnant lieu à des produits typiques (produits du terroir), de qualité irrégulière. Les résultats de Bufole (2014) ont révélé la préférence des consommateurs du «Mashanza» au regard de la provenance des différents produits trouvés sur le marché de Bukavu. L'insuffisance des travaux relatifs aux produits fermiers peut limiter la reconnaissance officielle de leur typicité (Mogessie Assenafi cité par Duteurtre, 2003). Malheureusement, tout comme d'autres fromages fabriqués dans les pays en développement, le «Mashanza», n'est pas suffisamment documenté, sa production étant demeurée empirique. Au vu de ce qui précède et tenant compte de l'intérêt qu'il présente, une connaissance plus approfondie est indispensable afin de combler les lacunes existant sur ce produit au regard des produits locaux fabriqués ailleurs.

\section{MATÉRIELS ET MÉTHODES}

Description du milieu d'étude : L'étude a été réalisée à partir du mois de juillet 2016 dans la province du SudKivu à l'est de la RD Congo, située entre $1^{\circ} 36^{\prime}$ et $5^{\circ}$ de latitude Sud et $26^{\circ} 47^{\prime}$ et $29^{\circ} 20^{\prime}$ de longitude Est. La température moyenne annuelle est de $19^{\circ} \mathrm{C}$, et l'altitude varie entre $773 \mathrm{~m}$ et $3.000 \mathrm{~m}$. On y trouve deux saisons à savoir, la saison sèche qui dure 3 mois, de juin à septembre, et la saison des pluies qui dure 9 mois (Ministère du Plan, 2005 ; Mutwedu et.al, 2015). Le recensement en 2002 du cheptel bovin de différentes races élevées était estimé à 170664 têtes (Ministère du Plan, 2011).

Echantillonnage et collecte des données: Cette étude a concerné précisément les territoires de Kabare, Kalehe, Uvira et Walungu, où la fabrication du fromage traditionnel «Mashanza» est connue et habituelle. L'enquête par questionnaire a été réalisée au mois de Juin 2016 auprès des unités de production du fromage frais «Mashanza» et a touché un total de 132 fabricants dont 20 à Kabare, 18 à Kalehe, 45 à Uvira et 49 à
En outre, la maîtrise de l'environnement de production et celle des paramètres technologiques des différents procédés pourront permettre d'étudier les voies d'amélioration et de standardisation de la qualité du produit fini. Ainsi, l'objectif de la présente étude est d'établir un diagnostic des procédés usuels de fabrication du fromage frais «Mashanza» produit à Kabare, Kalehe, Uvira et Walungu, dans la province du Sud-Kivu. De manière spécifique, l'étude vise à :

- caractériser les unités de production enquêtées ;

- décrire les procédés de fabrication du Mashanza suivis par les fermiers dans différentes zones ciblées ;

- présenter les caractéristiques du «Mashanza» telles que perçues par les fabricants dans les milieux d'étude.

Walungu. Le choix des unités enquêtées était déterminé par l'accessibilité et la fonctionnalité des unités de transformation ainsi que l'ancienneté dans l'activité. Le classement des unités de production a été réalisé sur base des critères ci-après: la taille, l'existence d'un local approprié, un minimum d'équipement de travail, la nature et la capacité des équipements de transformation, le nombre de salariés dans l'unité et la quantité de matière première transformée par opération..

Les informations recueillies ont concerné: le fabricant, les caractéristiques de l'unité de production, le choix des matières premières, la description des procédés de fabrication et des équipements utilisés et la qualité du « Mashanza » et du ferment.

Analyse des données : Les analyses des données de l'enquête ont été réalisées par la statistique descriptive en se servant des logiciels Excel et SPSS 20.

\section{RÉSULTATS ET DISCUSSION}

Caractérisation des personnes enquêtées : Les fabricants de «Mashanza» sont majoritairement des hommes. Ils représentent $100 \%$ des personnes enquêtées à Walungu, $95 \%$ à Kabare, $89 \%$ à Kalehe, excepté à Uvira où les femmes sont fortement impliquées, représentant à elles seules $86,7 \%$ contre $13,3 \%$ d'hommes. Elles s'occupent exclusivement de la transformation du lait en fromage frais et de la vente directe du « Mashanza ». 
Tableau 1: Quelques caractéristiques des personnes enquêtées

\begin{tabular}{|c|c|c|c|c|c|c|}
\hline & ation (territoire) & & Walungu & Kabare & Kalehe & Uvira \\
\hline & $\operatorname{ctif}(N): 132$ & & 49 & 20 & 18 & 45 \\
\hline & Homme & $\%$ & 100 & 95 & 88,9 & 13,3 \\
\hline Sexe & Femme & $\%$ & 0 & 5 & 11,1 & 86,7 \\
\hline & Total & & 100 & 100 & 100 & 100 \\
\hline & $10-19$ & $\%$ & 2,0 & 0 & 16,7 & 26,7 \\
\hline & $20-29$ & $\%$ & 2,0 & 15 & 27,8 & 31,1 \\
\hline & $30-39$ & $\%$ & 8,2 & 10 & 16,7 & 20 \\
\hline & $40-49$ & $\%$ & 14,3 & 20 & 11,1 & 6,7 \\
\hline Age & $50-59$ & $\%$ & 18,4 & 10 & 11,1 & 4,4 \\
\hline & $60-69$ & $\%$ & 6,1 & 0,0 & 5,6 & 4,4 \\
\hline & 70 et plus & $\%$ & 4,1 & 0,0 & 0 & 0 \\
\hline & Non défini & $\%$ & 44,9 & 45,0 & 11,1 & 6,7 \\
\hline & Total & & 100 & 100 & 100 & 100 \\
\hline & Aucun & $\%$ & 2,0 & 0,0 & 5,6 & 11,1 \\
\hline & Primaire & $\%$ & 8,2 & 0 & 11,1 & 13,3 \\
\hline Niveau d'études & Secondaire & $\%$ & 4,1 & 0 & 0 & 46,7 \\
\hline & Indéterminé & $\%$ & 85,7 & 100,0 & 83,3 & 28,9 \\
\hline & Total & & 100 & 100 & 100 & 100 \\
\hline & $1-5$ ans & $\%$ & 20,4 & 15,0 & 61,1 & 40,0 \\
\hline & $6-10$ ans & $\%$ & 14,3 & 5,0 & 5,6 & 20,0 \\
\hline $\begin{array}{l}\text { Experrence uaris } \\
\text { l'activitó }\end{array}$ & Plus de 10 ans & $\%$ & 34,7 & 30,0 & 11,1 & 33,3 \\
\hline & Sans réponse & $\%$ & 30,6 & 50,0 & 22,2 & 6,7 \\
\hline & Total & & 100 & 100 & 100 & 100 \\
\hline
\end{tabular}

Les tranches d'âge sont diverses et structurées comme suit: dans la classe d'âge de 10 à 19 ans, on a trouvé plus d'individus à Kalehe $(16,7 \%)$ et à Uvira $(26,7 \%)$; dans celle de 20 à 29 ans, on a $2 \%$ d'individus à Walungu, $15 \%$ à Kabare, $27,8 \%$ à Kalehe et $31,1 \%$ à Uvira; dans celle de 30 à 39 ans, la situation semble s'inverser et on a $14,3 \%$ d'individus à Walungu, $20 \%$ à Kabare, $11,1 \%$ à Kalehe et $6,7 \%$ à Uvira ; entre 50 et 59 ans, on a $18,4 \%$ d'individus à Walungu, $10 \%$ à Kabare, $11,1 \%$ à Kalehe et $4,4 \%$ à Uvira. Dans les classes suivantes, on trouve peu d'individus. Le taux de non réponse est de 27,3\%. Ainsi, les individus enquêtés sont plus âgés. La représentativité des différentes tranches d'âge reflète non seulement l'encrage de la tradition de la consommation du «Mashanza », mais aussi l'intérêt sur le plan socioéconomique de sa production pouvant générer des opportunités, d'autant plus qu'au Sud-Kivu le chômage est élevé. Selon un rapport du PNUD (2009), le chômage y est plus important $(9,9 \%)$ qu'au niveau national, avec un taux évalué à $3,7 \%$; le taux de chômage variant selon le milieu, de 6,9\% dans le milieu rural et $15,3 \%$ dans les cités. Concernant le niveau d'instruction, il n'a pas été révélé par plus de la moitié des enquêtés Cependant, les résultats ont montré que $2 \%$ à Walungu, $5,6 \%$ à Kalehe et $11,1 \%$ à Uvira n'ont pas fréquenté l'école; $8,2 \%$ à Walungu, $11,1 \%$ à Kalehe et $13,3 \%$ à Uvira ont fréquenté l'école primaire et enfin $4,1 \%$ à Walungu et $46,7 \%$ à Uvira ont atteint le niveau du secondaire. Cela se comprend puisque dans la plupart des fermes visitées, ce sont plus les travailleurs (gérants, techniciens chargés de la fabrication du «Mashanza») qui étaient présents lors de l'enquête. D'après le PNUD (2009), la Province du SudKivu compte près de $34,3 \%$ des non instruits contre $28,1 \%$ ayant atteint le niveau primaire, $35,8 \%$ le niveau secondaire et $1,5 \%$ le niveau universitaire. Par ailleurs, du point de vue expérience dans l'activité de fabrication de "Mashanza», les résultats ont révélé les taux les plus élevés à Walungu, Kabare et à Uvira. On note un taux élevé des répondants dans la classe de plus de 10 ans d'expérience à Walungu (34,76\%), Uvira $(33,3 \%)$, Kabare (30\%) et Kalehe $(11,1 \%)$, et dans la classe de 1 à 5 ans, on a plus d'enquêtés à Kalehe $(61,1 \%)$ suivi d'Uvira (40\%), de Walungu (20,4\%) et enfin de Kabare $(15 \%)$.

Caractérisation des unités de production et d'élevage : Les résultats obtenus ont montré que les 
fermes traditionnelles sont présentes à $100 \%$ à Kabare et Walungu, et $72,2 \%$ à Kalehe; les établissements artisanaux ou traditionnels représentent $93,3 \%$ à Uvira et les établissements semi-modernes, $27,8 \%$ à Kalehe. L'établissement artisanal ou traditionnel a été la caractéristique des producteurs d'Uvira où la majorité des enquêtés $(93,3 \%)$, essentiellement les femmes, achètent du lait auprès des fermiers et fabriquent le fromage frais pour être écoulé directement le long de la route Bukavu-Uvira par des marchands groupés à Bweghera, Sange et Luberizi.

Tableau 2: Caractéristiques des unités de production du «Mashanza» au Sud-Kivu

\begin{tabular}{|c|c|c|c|c|c|c|}
\hline \multicolumn{3}{|c|}{ Localisation } & Walungu & Kabare & Kalehe & Uvira \\
\hline \multicolumn{3}{|c|}{ Effectif: 132} & 49 & 20 & 18 & 45 \\
\hline \multirow{4}{*}{$\begin{array}{l}\text { Types d'unités de } \\
\text { production }\end{array}$} & Etablissement semi-moderne & $\%$ & 0 & 0 & 27,8 & 0 \\
\hline & \begin{tabular}{|l|} 
Ferme traditionnelle \\
\end{tabular} & $\%$ & 100 & 100 & 72,2 & 6,7 \\
\hline & Etablissement traditionnel & $\%$ & 0 & 0 & 0 & 93,3 \\
\hline & Total & $\%$ & 100 & 100 & 100 & 100 \\
\hline \multirow{6}{*}{$\begin{array}{l}\text { Nombre de personnes } \\
\text { impliquées dans } \\
\text { l'activité }\end{array}$} & $1-5$ & $\%$ & 95,9 & 90 & 83,3 & 97,8 \\
\hline & $6-10$ & $\%$ & 0 & 10 & 5,6 & 0 \\
\hline & \begin{tabular}{|l|}
$11-15$ \\
\end{tabular} & $\%$ & 2,0 & 0 & 0 & 2,2 \\
\hline & $16-20$ & $\%$ & 2,0 & 0 & 0 & 0 \\
\hline & indéterminé & $\%$ & 0 & 0 & 11,1 & 0 \\
\hline & Total & $\%$ & 100,0 & 100,0 & 100,0 & 100,0 \\
\hline \multirow{5}{*}{$\begin{array}{l}\text { Qualité des personnes } \\
\text { impliquées }\end{array}$} & Membres familles & $\%$ & 42,9 & 10 & 27,8 & 93,3 \\
\hline & Salariés & $\%$ & 38,8 & 85 & 66,7 & 4,4 \\
\hline & Ristourne & $\%$ & 18,4 & 5 & 5,6 & 0 \\
\hline & Autres & $\%$ & 0 & 0 & 0 & 2,2 \\
\hline & Total & $\%$ & 100,0 & 100,0 & 100,0 & 100,0 \\
\hline
\end{tabular}

Toutefois, il existe d'autres points de vente sur l'axe Bukavu-Uvira qui n'ont pas fait l'objet de la présente étude. A Uvira, cette tâche est réalisée par de nombreuses femmes qui transforment de petites quantités de lait qui peuvent être ainsi écoulées rapidement et générer des ressources pour la subsistance. Le taux de chômage étant élevé dans la province du Sud-Kivu, les études ont révélé que 90,8\% de la population du Sud-Kivu travaillent dans le secteur informel qui génère $95,2 \%$ des revenus des ménages. La quasi-totalité de la population vit par l'auto-emploi, situation qui concerne plus les hommes que les femmes (PNUD, 2009). Dans les 4 milieux étudiés, on distingue des unités employant 1 à 5 personnes selon les enquêtés et représentant $95,9 \%, 90 \%, 83,3 \%$ et $97,8 \%$ respectivement à Walungu, Kabare, Kalehe et Uvira; d'autres emploient 6 à 10 personnes $(10 \%$ à Kabare et $5,6 \%$ à Kalehe), 11 à 15 personnes ( $2 \%$ à Walungu et $2,2 \%$ à Uvira) ou encore 16 à 20 personnes ( $2 \%$ à Walungu). Etant donné que la plupart d'unités sont de type artisanal, on peut supposer que leur capacité de production est faible et ne nécessiterait pas par conséquent un personnel nombreux et qualifié. S'agissant de la qualité des personnes impliquées dans la gestion de l'unité de production du Mashanza, on distingue les membres de famille : $42,9 \%$ d'enquêtés à Walungu, $10 \%$ à Kabare, $27,8 \%$ à Kalehe et $93 \%$ à Uvira; quant aux salariés, ils représentent $38,8 \%$ à Walungu, $85 \%$ à Kabare, $66,7 \%$ à Kalehe et $4,4 \%$ à Uvira. Les prestations sous forme de ristourne sont rares, mais plus fréquentes à Walungu $(18,4 \%$ des enquêtés) qu'à Kabare $(5 \%)$ et Kalehe $(5,6 \%)$. D'autres formes de prestation existent à Uvira selon 2,2\% de personnes enquêtées.

\section{Fabrication du «Mashanza»}

Quantités de lait trait et transformé : Les quantités minimales produites sont variables d'un milieu à un autre, il en va de même des quantités transformées. La quantité de lait trait varie de 0,5 à $70 \mathrm{~L}$ à Walungu, de 0,5 à $120 \mathrm{~L}$ à Kabare, de 1 à $135 \mathrm{~L}$ à Kalehe et enfin de 2,5 à $30 \mathrm{~L}$ à Uvira. La quantité de lait transformé, varie de 2 à $70 \mathrm{~L}$ à Walungu, de 5 à $120 \mathrm{~L}$ à Kabare, de 5 à $135 \mathrm{~L}$ à Kalehe et 2,5 à $300 \mathrm{~L}$ à Uvira. Les quantités les plus fréquemment transformées sont de $10 \mathrm{~L}$ à 
Walungu, et de $20 \mathrm{~L}$ à Kabare, Kalehe et Uvira.

Tableau 3: Paramètres statistiques relatifs au lait trait et au lait transformé selon les enquêtés

\begin{tabular}{|l|c|c|c|c|c|c|c|c|}
\hline \multirow{2}{*}{ Paramètres } & \multicolumn{4}{c|}{$\begin{array}{c}\text { Quantité de lait transformée par opération } \\
\text { (litre) }\end{array}$} & \multicolumn{3}{c|}{ Quantité de lait trait par jour (litre) } \\
\hline & Walungu & Kabare & Kalehe & Uvira & Walungu & Kabare & Kalehe & Uvira \\
\hline Effectif & 49 & 20 & 12 & 41 & 49 & 20 & 12 & 41 \\
\hline Moyenne & 14,0 & 40,5 & 38,7 & 62,7 & 6,4 & 30,6 & 29,5 & 19,7 \\
\hline Ecart-type & 15,77 & 39,14 & 47,50 & 76,28 & 11,35 & 36,62 & 50,15 & 11,37 \\
\hline Mode & 10 & 20 & 20 & 20 & 1 & 20 & 1 & sans \\
\hline Minimum & 2 & 5 & 5 & 2,5 & 0,5 & 0,5 & 1 & 2,5 \\
\hline Maximum & 70 & 120 & 135 & 300 & 70 & 120 & 135 & 30 \\
\hline & & & & & & & & \\
\hline & & & & & & & & \\
\hline
\end{tabular}

Ratio ferment/lait : Le lait de vache et le lactosérum, ferment communément appelé Magunjo dans la langue locale «Shi», sont les principales matières premières. De manière générale, le rapport $1 / 2$ est utilisé par $4,1 \%$ des fabricants à Walungu et à Uvira particulièrement $84,4 \%$ des fabricants sont concernés. Les raisons ne sont pas connues, mais on peut avancer l'idée que bien que la fabrication de "Mashanza » étant un mode de vie pour la majorité des enquêtés, ces derniers cherchent à accélérer la vitesse de fermentation afin d'écouler rapidement leur production, ce qui nécessite l'usage d'une quantité élevée de ferment. D'autres rapports $1 / 10,2 / 10$ et $3 / 10$ sont également utilisés. Par contre, $32,7 \%$ à Walungu, $50 \%$ à Kabare, $83,3 \%$ à Kalehe et $4,4 \%$ à Uvira utilisent d'autres rapports laitferment. II s'agit des proportions suivantes : 3 parties de ferment contre 4 de lait, voire même 5 parties de lait (3/4 ou 3/5); d'une fermentation continue durant laquelle le lait est ajouté progressivement sur le «Mashanza» ou encore la fermentation spontanée. Le taux de non réponses a été de 5,3\%.

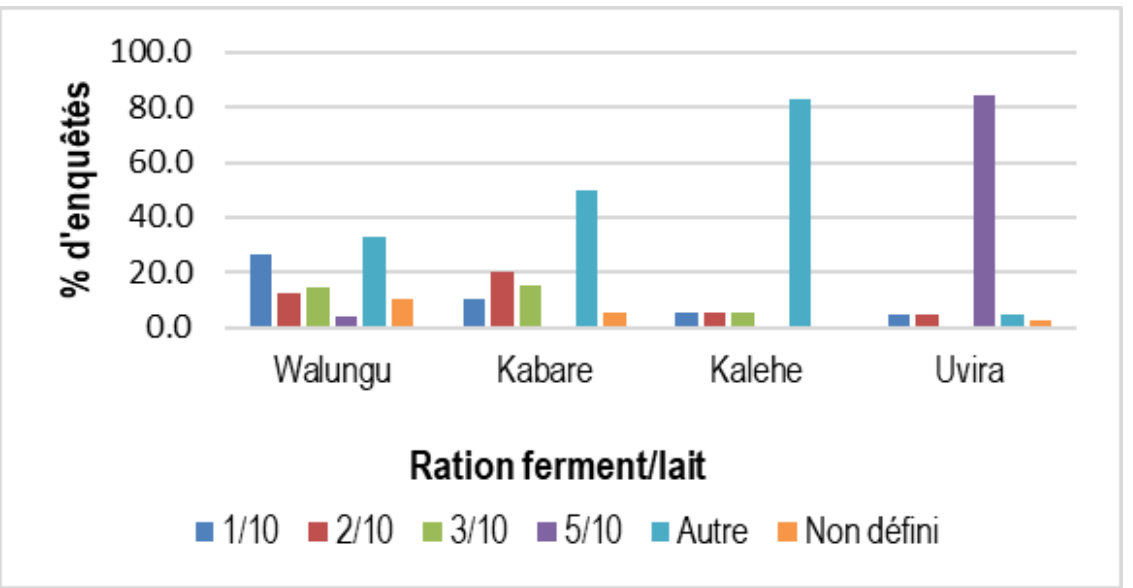

Figure 2 : Ratio ferment/lait employé lors de l'inoculation dans la fabrication du «Mashanza»

Rendement technologique : Le rendement fréquent est de $20 \mathrm{Kg}$ pour 100 litres de lait transformé dans les 4 territoires avec des moyennes de $24 \pm 11 \mathrm{Kg}$ à Kabare, $24 \pm 10$ à Kalehe, $21 \pm 8$ à Uvira et $23 \pm 6$ à Walungu. La diversité des procédés pourrait justifier, en partie, les écarts des rendements entre fabricants. Le rendement fromager correspond à un poids de fromage fabriqué à partir d'une quantité connue de lait, et rapporté à $100 \mathrm{~L}$ ou $100 \mathrm{~kg}$ de matière première (Goudédranche et al., 2001). II est essentiellement influencé par la teneur en protéines coagulables, mais les facteurs inhérents à la qualité de lait et aux modalités de transformation en fromage peuvent l'influencer également (Cuvillier, 2005). Les valeurs moyennes pour les pâtes fraîches se rangent entre 25$35 \mathrm{~kg}$ (Ramet et al., 1985). Les valeurs trouvées ici par 
les enquêtés sont proches de celles données dans la littérature.

Procédés de fabrication: Les procédés inventoriés sont au nombre de 5 (figures 2 et 3). Le plus fréquent et adopté par la majorité d'enquêtés dans tous les 4 territoires est le procédé 1 ; il concerne 93,9\% d'individus à Walungu, $85 \%$ à Kabare, $38,9 \%$ à Kalehe et $97,8 \%$ à Uvira. Ce procédé se caractétise par la succession d'étapes suivantes: "Traite-Collecte-Mise en cuve -Inoculation-Fermentation-EgouttageConditionnement». La filtration se fait simultanément avec la collecte ou la traite.

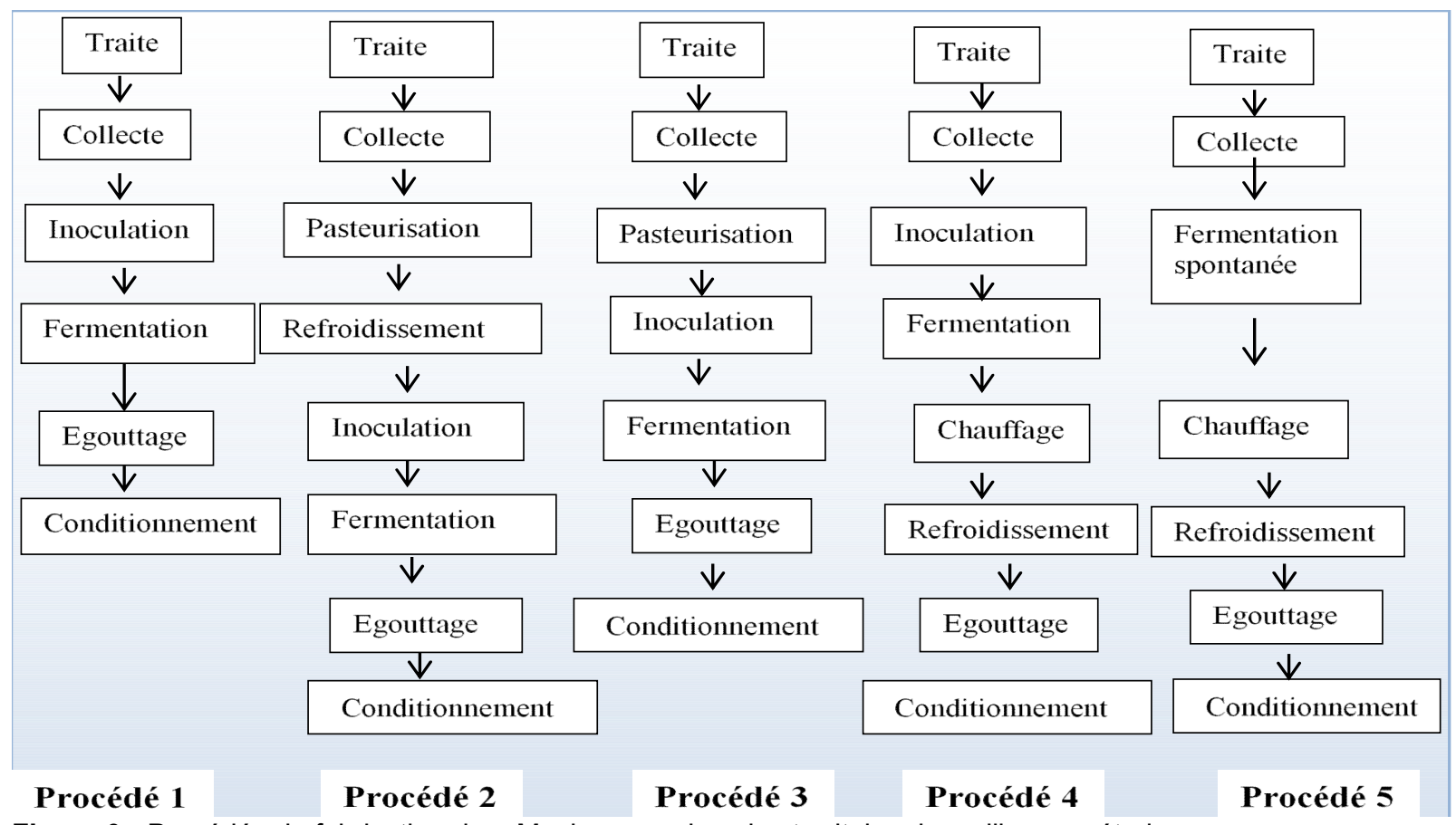

Figure 3 : Procédés de fabrication du « Mashanza » dans les territoires les milieux en étude
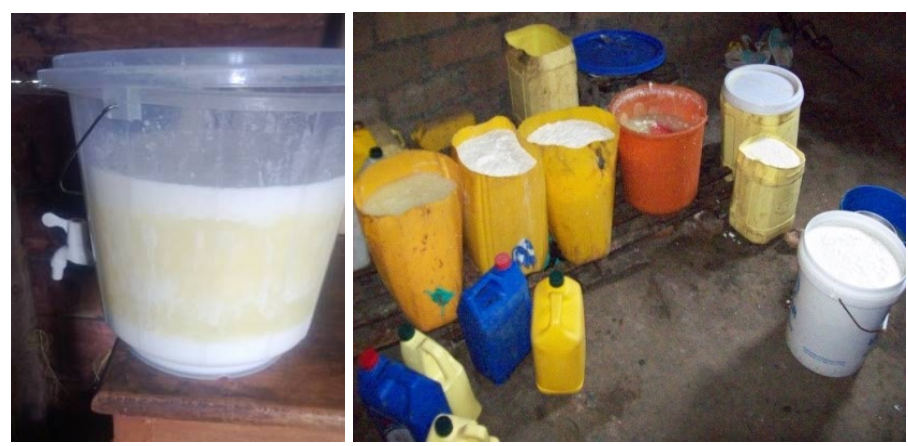

Figure 4 : Cuves de fermentation du « Mashanza » : de gauche à droite, seau avec couvercle et vanne d'égouttage du lactosérum, bidons découpés et seaux sans couvercles 


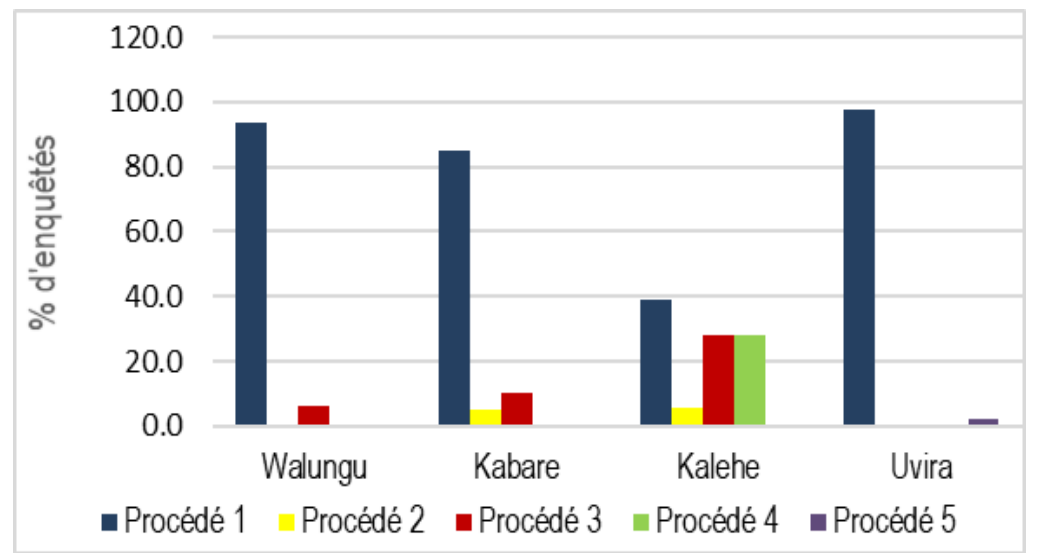

Figure 5 : Procédés suivis par les fabricants de Mashanza dans les 4 territoires

Traite et collecte du lait : Les récipients utilisés pour la traite sont soient les cruches en plastique ou en bois et les seaux, munis d'un tamis filtrant est observé, tandis que pour la collecte du lait, les bidons en plastique sont courants.

Traitement thermique du lait : La durée du traitement thermique et son intensité restent vagues, seules les indications telles que début d'ébullition ou formation d'une écume ont été mentionnées. Or pour assainir le lait, il est souvent pratiqué un traitement thermique plus ou moins intense de l'ordre de 63 à $95{ }^{\circ} \mathrm{C}$ pendant 4 à 5 min pour des fromages de type pâte fraîche (Goudedranche et al., 2001).

Inoculation et fermentation: Le ferment est habituellement conservé soit dans un bidon, soit dans une calebasse de petite dimension appelé "Kahunguliro» en langue Shi. II est bouché par un couvercle à base de sachet plastique pour assurer son adhérence. La durée d'inoculation, dans la plupart des cas, n'excède pas 10 minutes. Pour la fermentation, une autre calebasse de dimension moyenne appelée dans la langue "shi» Nshuha est employée. Dans d'autres unités de fabrication, des seaux et tanks avec couvercle munis de robinet allant jusqu'à $100 \mathrm{~L}$ sont utilisés (fig. 4). A Bweghera, les seaux et bidons découpés sont d'usage. Quant à la durée de la fermentation, le mode est de 24 heures dans tous les milieux avec une durée maximale pouvant atteindre 72 heures à Kabare et 96 heures à Kalehe et Walungu. Ces durées élevées se justifient en cas de fermentation continue pour assurer la conservation du Mashanza.

Egouttage: Les seaux munis de vannes ou robinet sont utilisés dans les fermes traitant les quantités élevées de mashanza (figure 4). Dans d'autres unités, une tige de bambou, une cuillère ou encore une assiette permettent d'évacuer le lactosérum. Comme critère de la fin d'égouttage, les enquêtés ont mentionné l'absence de ressuyage du lactosérum et le timing.

Conditionnement: D'après les données collectées, la conservation par réfrigération est rare $(2 \%)$ au niveau des unités de fabrication. A Kabare, Kalehe et Walungu, le mode prédominant est le seau avec couvercle tandis qu'à Uvira, le produit est conditionné dans des assiettes déposées dans un bassin. Le sachet plastique est utilisé pour conditionner le produit prêt à la vente. La figure 5 donne une diversité des types et modes de conditionnement utilisés pour le Mashanza dans les 4 territoires. 


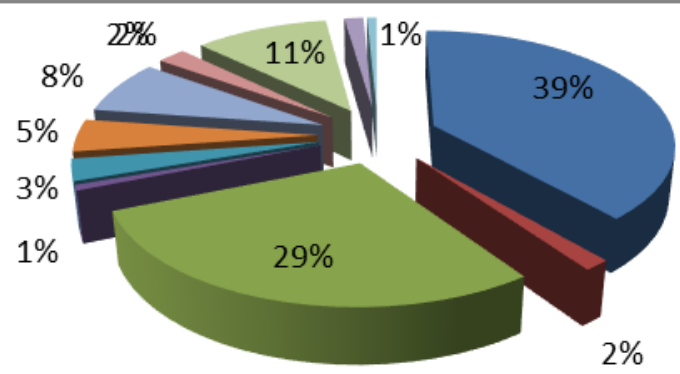

$\begin{array}{lll}\text { neau avec couvercle } & \text { frigo } & \text { assiette dans bassin } \\ \text { Dans le lactosérum } & \text { assiette } & \text { sachet plastique } \\ \text { autre } & \text { seau } & \text { Non déterminé } \\ \text { aucun emballage } & \text { sans commercialisation } & \end{array}$

Figure 6 : Pourcentage d'enquêtés selon les types et modes de conditionnement du Mashanza

Qualité organoleptique du Mashanza

Tableau 4 : Pourcentage d'enquêtés selon les paramètres qualitatifs du «Mashanza»

\begin{tabular}{|c|c|c|c|c|c|c|}
\hline \multirow{3}{*}{$\begin{array}{l}\text { Paramètres } \\
\text { Descripteurs }\end{array}$} & \multirow{3}{*}{$\begin{array}{r}\text { Modalités } \\
\text { Effectif : } 132\end{array}$} & & \multicolumn{4}{|c|}{ Localisation } \\
\hline & & & Walungu & Kabare & Kalehe & Uvira \\
\hline & & & 44 & 17 & 18 & 42 \\
\hline \multirow{8}{*}{ Goût } & neutre & $\%$ & 46,9 & 45,0 & 11,1 & 6,7 \\
\hline & aigre & $\%$ & 4,1 & 5,0 & 22,2 & 11,1 \\
\hline & Autre (non défini) & $\%$ & 0,0 & 5,0 & 0,0 & 2,2 \\
\hline & sucré & $\%$ & 2,0 & 0,0 & 0,0 & 24,4 \\
\hline & doux & $\%$ & 44,9 & 35,0 & 16,7 & 2,2 \\
\hline & Non réponse & $\%$ & 2,0 & 10,0 & 50,0 & 46,7 \\
\hline & intermédiaire entre sucré et aigre & $\%$ & 0,0 & 0,0 & 0,0 & 6,7 \\
\hline & Total & $\%$ & 100,0 & 100,0 & 100,0 & 100,0 \\
\hline \multirow{5}{*}{ Couleur } & Blanche & $\%$ & 89,8 & 85,0 & 100,0 & 93,3 \\
\hline & Blanc-crème & $\%$ & 2,0 & 10,0 & 0,0 & 6,7 \\
\hline & jaunâtre & $\%$ & 8,2 & 0,0 & 0,0 & 0,0 \\
\hline & indéterminé & $\%$ & 0,0 & 5,0 & 0,0 & 0,0 \\
\hline & Total & $\%$ & 100,0 & 100,0 & 100,0 & 100,0 \\
\hline \multirow{6}{*}{ Odeur } & normal & $\%$ & 81,6 & 60,0 & 16,7 & 22,2 \\
\hline & fermenté & $\%$ & 2,0 & 0,0 & 0,0 & 4,4 \\
\hline & autre & $\%$ & 6,1 & 0,0 & 0,0 & 0,0 \\
\hline & indéterminé & $\%$ & 10,2 & 40,0 & 77,8 & 71,1 \\
\hline & odeur de lait & $\%$ & 0,0 & 0,0 & 5,6 & 2,2 \\
\hline & Total & $\%$ & 100,0 & 100,0 & 100,0 & 100,0 \\
\hline \multirow{5}{*}{ Consistance } & molle & $\%$ & 2,04 & 0,00 & 0,00 & 0,00 \\
\hline & \begin{tabular}{|l|} 
ferme \\
\end{tabular} & $\%$ & 75,51 & 55,00 & 5,56 & 6,67 \\
\hline & intermédiaire & $\%$ & 22,45 & 45,00 & 88,89 & 93,33 \\
\hline & indéterminé & $\%$ & 0,00 & 0,00 & 5,56 & 0,00 \\
\hline & Total & $\%$ & 100,00 & 100,00 & 100,00 & 100,00 \\
\hline
\end{tabular}


Au vu des résultats ci-dessus, on peut noter ce qui suit: i. $\quad$ En rapport avec la perception du goût du «Mashanza», à Walungu, 46,9\% lui trouvent un goût neutre, $44,9 \%$ une saveur douce, $4,1 \%$ une saveur aigre, $2 \%$ un goût sucré, $2 \%$, une saveur indescriptible. A Kabare, $45 \%$ lui trouvent un goût neutre, $35 \%$ une saveur douce, $5 \%$ une saveur aigre, $10 \%$, une saveur indescriptible et enfin $5 \%$ ont du mal à définir le goût. Enfin à Kalehe et Uvira, les enquêtés ont trouvé respectivement un goût neutre (11,1 et $6,7 \%)$, un goût aigre (22,2 et $11,1 \%)$, un goût sucré ( 0 et $24,4 \%$ ), une saveur douce $(16,7$ et $2,2 \%)$, une saveur intermédiaire entre sucrée et aigre ( 0 et $6,7 \%$ ). Seulement $2,2 \%$ ont eu du mal à définir le goût du Mashanza à Uvira. Le taux de non réponse est tès élevé à Kabare, Kalehe et Uvira. Selon FAO (1998), la pâte obtenue en fin d'égouttage se caractérise par une forte humidité, un $\mathrm{pH}$ bas $(4-4,2)$ qui lui confère son goût acidulé et une faible minéralisation $(0,1 \%$ de calcium, $0,2 \%$ de phosphore). Elle contient encore, sous forme d'acide lactique, environ $25 \%$ du lactose du lait. Par ailleurs, pour Gorban et Izzeldin (1997), le pH et le goût du lait peuvent dépendre de la nature des fourrages et de la disponibilité de l'eau.

ii. En rapport avec la couleur du «Mashanza», elle est perçue par la majorité des fabricants $(89,8 \%$ à Walungu, $85 \%$ à Kabare, $100 \%$ à Kalehe et $93,3 \%$ à Uvira) comme étant blanche, d'autres le trouvent soit blanc-crème ( $2 \%$ à Walungu, $10 \%$ à Kabare et $6,7 \%$ à Uvira) et seulement $8,2 \%$ à Walungu le trouvent jaunâtre; enfin $5 \%$ ne se sont pas prononcés. Park et al (1983); cité par Coulon et al (2005) rapportent l'effet du Carotène présent en grandes quantités dans les fourrages verts, sur la coloration jaune des produits laitiers.

iii. Quant à l'odeur du «Mashanza, la majorité des fabricants perçoivent le mashanza comme ayant une odeur normale ( $81,6 \%$ à Walungu, $60 \%$ à Kabare, $16,7 \%$ à Kalehe et $22,2 \%$ à Uvira. D'autres lui trouvent une odeur de fermenté ou une odeur de lait. Une grande part des fabricants ne se sont pas déterminés sur leur perception. En effet, les perceptions varient d'un individu à un autre et résulteraient des différences interindividuelles de sensibilité ou de l'acceptabilité qui existent entre sujets (Depledt et Sauvageot, 2006). iv. Enfin, en rapport avec la consistance, les résultats ont révélé que la majorité des fromages produits dans les quatre entités sont classés dans la catégorie à consistance ferme, et intermédiaire. Ainsi, compte tenu de la diversité des procédés, certaines étapes pourraient expliquer les différences perçues par les consommateurs et fabricants du point de vue consistance ou tout simplement fermeté du fromage. II peut s'agir, notamment de la coagulation lors de la fermentation, de l'égouttage (durée et modes d'égoutage), aptitude du gel à évacuer le lactosérum (Ramet, 1985), ou suite au chauffage ou thermisation $\mathrm{du}$ lait. Ces auteurs soulignent que la friabilité du fromage dépend des facteurs technologiques variés: un excès d'acidification, une activité protéolytique élevée ou un chauffage élevé du lait ; ce qui est confirmé par Jeantet et ses collaborateurs (2008) en montrant que les cinétiques d'égouttage et d'acidification contribuent à la diversité des fromages. Par ailleurs, la teneur élevée en eau et le faible degré de minéralisation entraînent un manque de tenue et de cohésion du fromage (FAO, 1998).

Durée de vie du «Mashanza» et du ferment: La durée de conservation est estimée à moins d'une semaine jusqu'à une semaine par la majorité des enquêtés dans tous les territoires. Ce qui a été confirmé par une étude de FAO (1990). La durée de conservation pourrait dépendre, entre autres, du procédé utilisé, des conditions écologiques, et des modes de conditionnement adoptés. D'après Ramet (1985), les pâtes fraîches ont des valeurs de pH variant de 4,3 à 4,5 et d'activité de l'eau de 0,980 à 0,995 à la fin de l'égouttage et ne sont pas suffisamment basses pour assurer une stabilisation complète du substrat. Quant à la durée de conservation du ferment, elle est variable selon les milieux et varie de moins d'une semaine à 1 mois, voire même plus selon les enquêtés interrogés. Pourtant d'après FAO (1998), le sérum ensemencé en bactéries lactiques et autres microorganismes n'est pas stable et nécessite d'être réfrigéré, après pasteurisation, vers 5 à $8^{\circ} \mathrm{C}$. 


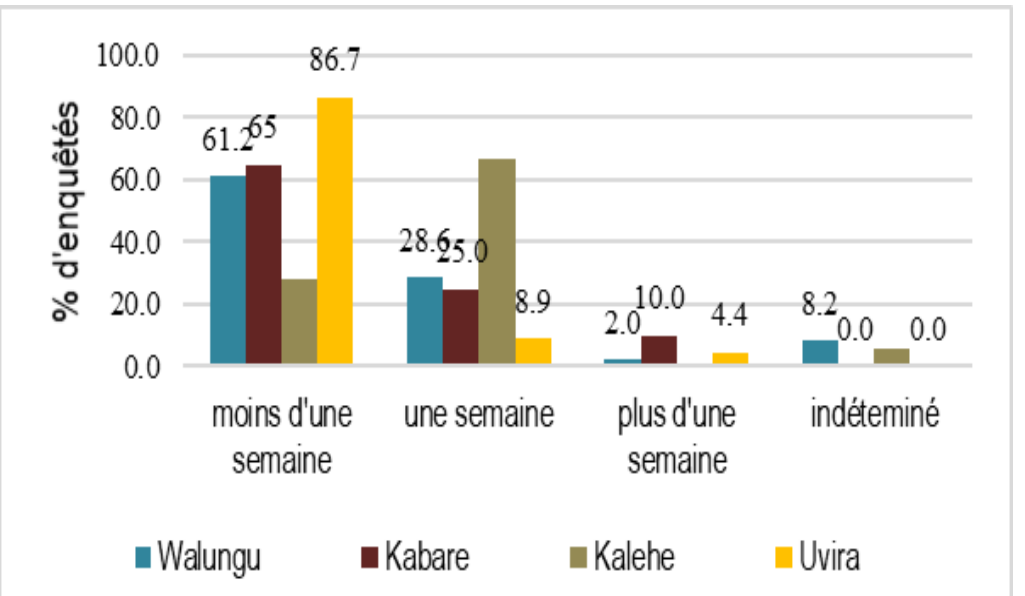

Figure 7 : Estimation de la durée de vie du Mashanza en \% par les enquêtés

\section{CONCLUSION ET PERSPECTIVES}

Ce travail avait pour objectif de réaliser un diagnostic des procédés de fabrication du «Mashanza». Au total, 5 procédés différents ont été inventoriés. Les étapes se succèdent comme suit: traite du lait - collecte (mise en cuve) - inoculation - fermentation - égouttage et conditionnement. Les variantes étant le filtrage et la thermisation du lait, le chauffage du lait après fermentation et l'égouttage (modes et durées). Ainsi, les résultats montrent l'existence des variabilités au niveau de la qualité des matières premières, du produit fini et des procédés en général, soit en termes

\section{REMERCIEMENTS}

Les auteurs remercient le VLIR pour son soutien financier ainsi que Patrick SAFARI, Jean-Charles

\section{RÉFÉRENCES BIBLIOGRAPHIQUES}

Bouraoui, A., 2014. Intérêt de fabrication de fromage analogue. http://www.memoireonline.com 104/14/8819/Intert-de-fabrication-de-fromageanalogue.html, (2/4/2017).

Bufole, N., 2014, Contribution à la connaissance de la consommation de Mashanza dans la ville de Bukavu. «Mémoire: Université Catholique de Bukavu (Bukavu) », inédit, 27 pages.

Coulon, J.B., Delacroix-Buchet, A. Martin, B. et Pirisi, A., 2005. Facteurs de production et qualité sensorielle des fromages, INRA, Prod. Anim., 18 (1), 49-62. HYPERLINK "http://prodi nra.inra.fr/ft?id=1A5FCB46-340C-431A-A1F94B00F4F122D9" http://prodinra.inra.fr/ft?id $=1$ A5FCB46-340C-431A-A1F9-4B00F4F122 D9, (20/02/2017).

Cuvillier, D., 2005. Améliorer le rendement fromager. d'étapes, soit en termes de durées des différentes étapes, soit en termes des conditions de fabrication et d'équipement utilisés. La variabilité de la qualité et du rendement fromager milite pour un contrôle tout le long de la chaîne d'approvisionnement et de fabrication afin de garantir la qualité du produit et la sécurité des consommateurs. Les résultats obtenus peuvent aider d'une part, les chercheurs dans l'optimisation des procédés et de la qualité et l'identification des risques et d'autre part, orienter les fabricants dans le choix des meilleurs procédés et des pratiques d'hygiène.

CIRHUZA et Claude MUPOLE pour leur participation à la collecte des données de l'étude.

http://cfbourgogne.free.fr/IMG/pdf/fiche_amelio rer_rendement.pdf, consulté le 01/2/2019.

Depledt, F. et Sauvageot, F., 2002. Évaluation sensorielle des produits alimentaires. Technique de l'ingénieur, traité Agroalimentaire, F4000 v1, 7 Pages.

Duteurtre, G., 2003. Normes exogènes et traditions locales : la problématique de la qualité dans les filières laitières africaines. Rapport du séminaire "Lait Sain pour le Sahel», du 24 février au 1er mars 2003, Bamako. HYPERLINK "http://agritrop-prod.cirad.fr/51 7250/1/document_517250.pdf" http://agritropprod.cirad.fr/517250/1/document_517250.pdf, 13 Pages, (15/03/2017).

FAO, 1990. The technology of traditional milk products in developing countries. HYPERLINK " 
http://www.fao.org/docrep/003/t0251e/T0251E 00.htm\#TOC, 223 Pages, (15/03/2017).

FAO, 1998. Le lait et les produits laitiers dans la nutrition humaine. http://www.fao.org/docrep /t4280f/T4280FOf.htm\#Chapitre\%206\%20From ages, 255 Pages, (15/3/2017).

Gorban, A.M.S. and Izzeldin, O.M., 1997. Mineral content of camel milk and colostrum. L'effet du Changement Climatique sur l'élevage et la gestion durable des parcours dans les zones arides et semi-arides du Maghreb, J. Dairy Techn., 64, 471-474

Goudédranche, H., Camier-B., Gassi, J-Y et Schuck, P. 2001. Procédé de transformation fromagère (partie 1). Technique de l'ingénieur, traité Agroalimentaire, F6305. 11 Pages.

Jeantet, R, Croguennec, T., Schuck P.et Brulé, G. Brulé, G. 2008. Science des aliments. Biochimie - Microbiologie - Procédés Produits. Technologie des produits alimentaires, Tome 2. Paris: Technique \& Documentation, 456 Pages.

Kora, S. 2005. Lait et fromage au Bénin. Http://www.memoireonline.com/04/10/3418/m lait-et-fromage-au-Benin.html, $\quad(8 / 6 / 2017)$ Pages?

Mechai, A., Debabza, M. and Kirane, D., 2014. Screening of technological and probiotic properties of lactic acid bacteria isolated from Algerian traditional fermented milk products, International Food Research Journal 21(6): 2451-2457

Ministère du Plan, 2005. Monographie de la province du Sud- Kivu, PNUD-UNOPS, 179 Pages.

Ministere du Plan, 2011. Document de la Stratégie de Croissance et de Réduction de la

Pauvreté 2011-2015 de la Province du Sud-Kivu.104 pages.

Mutwedu, V.B , Ayagirwe, R. B.B., Mètre, K.T., Mugumaarhahama, Y., Sadiki, J.M.et Bisimwa, E.B., 2015. Systèmes de production cunicole en milieu paysan au Sud-Kivu, est de la RD Congo, Livestock Research for Rural Development 27 (10), http://www.lrrd.org /lrrd27/10/mutw27206.html, (17/5/2017).

PNUD, 2009, Pauvreté et conditions de vie des ménages, http://www.cd.undp.org/content/ dam/dem_rep_congo/docs/povred/UNDP-CD-

Profil-PROVINCE-Sud-Kivu.pdf? download, 19 Pages, (15/5/207)

Prajapati, J.B, 2011. Traditional Dairy Products in
Developing Countries. Dairy products: a healthy choice, IDFDairysummit. http://www.wds2011.com/Files/media/PrivateS pace/Presentations/IDFDairyProductsAHealthy ChoiceSunday/16.00---16.25---JB-Prajapati--Traditional-dairy-rev.pdf, 73 Pages, (8/11/2015).

Ramet, J.P., 1985. La fromagerie et les variétés de fromages du bassin Méditerranéen. Etude FAO. Production et santé animale (48). http://www.fao.org/docrep/004/X6551F/X6551 F00.HTM, 187 Pages (15/5/2017).

Tchobo, F. P., Ahouannou, C., Amegnoin, A., Mazou, M., Alitonou, G. A., Sohounhloue, D. C., \& Soumanou, M. M., 2014. Physico-chemical and microbiological characteristics of dried Waragashi. Academic Journals, 8(8), 447-455. 